\title{
More motivated but equally good: no effect of gamification on visual working memory
}

\section{performance}

Maria Mystakidou \& Ronald van den Berg

\section{ABSTRACT}

Gamification refers to the introduction of gaming elements such as scores and leaderboards in nongaming contexts. While there is growing evidence that gamification has positive effects on intrinsic motivation and engagement, it is largely unknown whether these effects translate to improved cognitive performance. Here, we examine whether gamification affects performance on a visual working memory (VWM) task. In Experiment 1, we gamified a standard delayedestimation task by introducing scores and a leveling system. On each trial, the subject's estimation error was mapped to a score between -100 and +100 and added to their total score. Subjects started at a set size of 1 and "leveled up" to the next set size each time they had accumulated 1,500 points. Post-experiment questionnaire data confirmed that subjects who performed the gamified version of the task were more motivated than control subjects. However, we found no difference in VWM performance between these two groups, nor between below-median and above-median motivated subjects. In Experiment 2, we tested for effects of trial-by-trial manipulations of motivation on VWM performance, by varying the scoring function across trials. Three scoring functions were used, with maxima of 7, 21, and 101 points. At the beginning of each trial, the subject was informed whether the potential reward was "low", "medium", or "high". Post-questionnaire data showed that subjects were more motivated on high-reward trials. However, we found no evidence for a difference in performance between the three reward levels. Our results suggest that gamification increases people's motivation to carry out visual working memory tasks, but it does not necessarily increase their performance.

Keywords: visual working memory; gamification; motivation; resource rationality; working memory capacity; cognitive constraints 


\section{INTRODUCTION}

There has recently been a surge in research on effects of "gamification", i.e., the use of gaming elements in non-gaming contexts such as learning, education, and marketing (e.g., (Deterding, Dixon, Khaled, \& Nacke, 2011; Dicheva, Dichev, Agre, \& Angelova, 2015; Hamari \& Lehdonvirta, 2010; Hanus \& Fox, 2015; Subhash \& Cudney, 2018)). Empirical work has suggested that gamification has positive effects on a variety of psychological outcomes, such as intrinsic motivation, enjoyment, engagement, and perceived competence (see Hamari et al., 2014 for a review). It is largely unknown, however, whether these effects are accompanied by improved cognitive performance. In the present study, we examine whether gamification improves people's performance on a visual working memory (VWM) task.

A prerequisite for finding effects of gamification on VWM performance is that allocation of VWM resources must be flexible - if it is fixed, no experimental manipulation can increase or decrease VWM performance. While there has been extensive research on describing VWM limitations (Brady, Konkle, \& Alvarez, 2011; Luck \& Vogel, 2013; Ma, Husain, \& Bays, 2014), few studies have asked why there are limitations in the first place. One possible answer to this question is that the sustained energy that is required to keep a memory alive (Fuster \& Alexander, 1971) induces a metabolic cost (Attwell \& Laughlin, 2001; Laughlin, 2001; Sterling \& Laughlin, 2015). In the presence of such a cost, a rational system would use its resources sparingly: resources should only be invested insofar the induced cost is compensated for by expected task reward. We recently formalized this idea in a resource-rational theory of VWM (Van den Berg \& Ma, 2018) and found that a model derived from this theory accounts well for earlier documented effects of set size (Bays, Catalao, \& Husain, 2009; Van den Berg, Shin, Chou, George, \& Ma, 2012; Wilken \& Ma, 2004) and item importance (Bays, 2014; Emrich, Lockhart, \& Al-Aidroos, 2017) on encoding precision. Inspired by these findings, we proposed that resource-rationality may be a general theory of how the brain allocates VWM resources and that VWM "limitations" are the result of a cost-benefit trade-off rather than a hardwired constraint on capacity. A key implication of this theory is that VWM resource allocation may be much more flexible than assumed so far.

If the amount of VWM resource utilized to a task is flexible, we may expect a relation between a subject's level of motivation and the amount of resource they invest in a task. Therefore, we hypothesize that performance on VWM tasks can be improved by gamifying the tasks. We test 
61 compare VWM performance between subjects who perform a standard VWM task and subjects

62 who perform a gamified version of that task. Experiment 2 uses a within-subject design, in which

63 the number of points that a subject can earn varies across trials. To preview our results, we find

64 that gamification increases motivation in both experiments, but we find no effect on VWM

65 performance.

\section{EXPERIMENT 1}

\subsection{Sharing of data and analysis scripts}

Data and Matlab scripts related to this experiment are available at https://osf.io/gb2kd/.

\subsection{Participants}

A total of 62 participants with self-reported normal or corrected-to-normal vision were recruited using posters at various campuses of Uppsala University (Table 1). The first 40 participants were randomly divided into the first three gamified groups. The remaining 22 participants were recruited later and randomly divided into the control group and the last gamified group. The study was approved by the Regional Ethical Review Board in Uppsala and conducted according to the Declaration of Helsinki Principles. All participants signed informed consent and received a cinema voucher with a value of approximately $\$ 12$ for their participation.

81 Table 1. Overview of participants. Eo refers to the estimation error (in degrees) at which the 82 scoring function mapped to a score of 0 points (see Figure 1B).

\begin{tabular}{cccccc}
\hline Condition & $\boldsymbol{E}_{\mathbf{0}}$ & Number of subjects & Age range & Mean age & Females \\
\hline Gamified, difficulty 1 & 60 & 13 & $20-47$ & $28.0 \pm 0.5$ & 7 \\
Gamified, difficulty 2 & 45 & 13 & $19-36$ & $24.4 \pm 0.4$ & 9 \\
Gamified, difficulty 3 & 30 & 14 & $20-43$ & $26.4 \pm 0.5$ & 10 \\
Gamified, difficulty 4 & 20 & 10 & $21-38$ & $25.2 \pm 0.5$ & 6 \\
Control & - & 12 & $20-35$ & $24.6 \pm 0.4$ & 9 \\
\hline
\end{tabular}




\subsection{Stimuli and materials}

Stimuli were presented on a 23" LCD screen at a resolution of $1920 \times 1080$ pixels in a dimly lighted room ${ }^{1}$. Subjects were seated at a distance of approximately $60 \mathrm{~cm}$ from the screen. Two centrally located concentric circles were used as a fixation point. Stimuli were dark gray ellipses, presented on a light gray background (Figure 1A). The ellipses had an area of 1,000 pixels ${ }^{2}$ and an eccentricity (elongation) of 0.95 . The stimuli were presented at a centrally located, invisible circle with a radius of 220 pixels. The location of the first stimulus was drawn randomly and all other stimuli were positioned such that equal spacing was ensured between any two neighboring stimuli. The orientation of each stimulus was drawn from a uniform distribution on the circle, with the constraint that the minimum circular distance (in degrees) between any two stimuli was at least $90 / N$, where $N$ indicates the set size. This constraint was intended to discourage subjects from using chunking strategies during encoding (Nassar, Helmers, \& Frank, 2018). Eye movements were recorded using a Tobii $4 \mathrm{C}$ eye tracker.

A

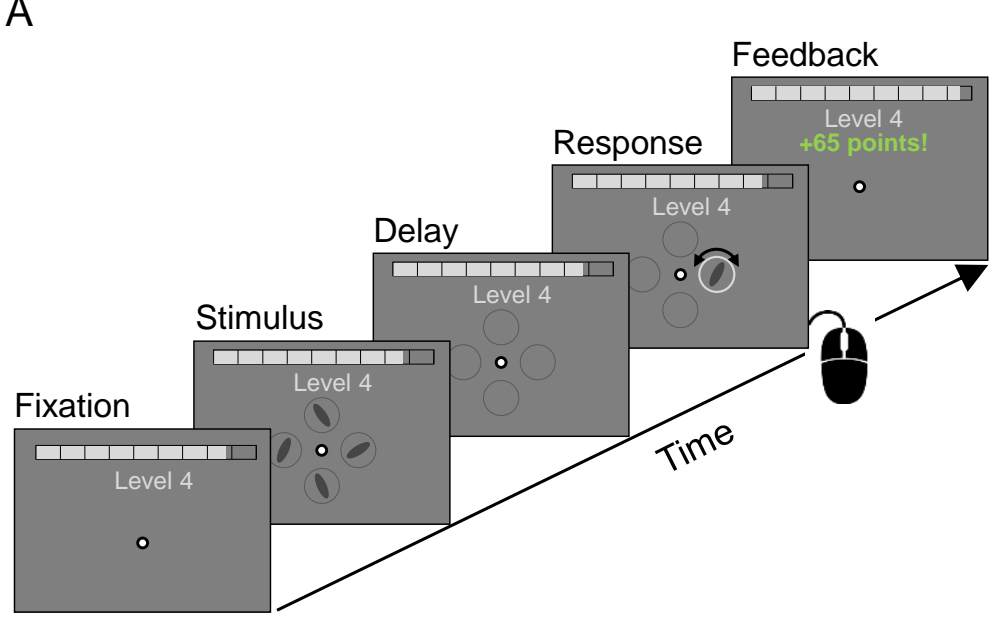

B

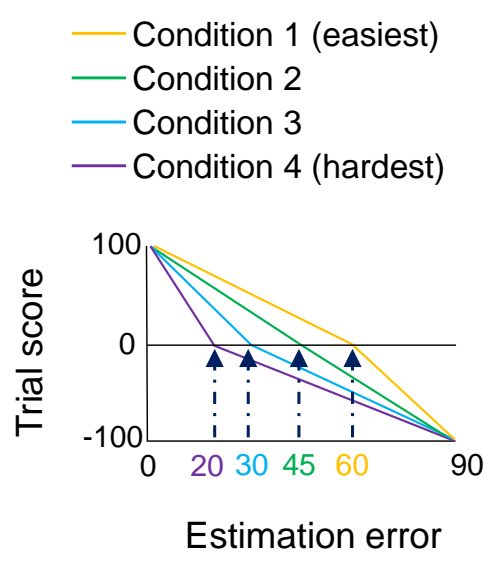

Figure 1 | Design of Experiment 1. Schematic illustration of a single experimental trial. (B) Scoring functions used in the four gamified conditions.

\footnotetext{
${ }^{1}$ The room contained two ceiling-mounted fluorescent lamps. The one farthest away from the experimental setup was turned on and the other on was turned off. However, a number of subjects reported that the lamp closest to the setup had spontaneously turned on during the experiment, possibly due to a technical error in the lamp's motion detector. Moreover, one subject was accidentally tested in a completely darkened room.
} 


\subsection{Task}

Subjects performed a delayed estimation task (Wilken \& Ma, 2004) with stimulus orientation as the relevant feature. Each trial started with a central fixation mark which the subject had to fixate at for at least $600 \mathrm{~ms}$. If a subject failed to fixate within 1 second, a message would appear on the screen telling the subject to "Please fixate at the central dot". After successful fixation, the subject was presented with $N$ oriented ellipses (200 ms). After a memory delay (1.5 s), the location of one ellipse was highlighted with a dashed circle. The task of the subject was to reproduce the orientation of the earlier displayed ellipse at that location. In order to respond, the subject would first click anywhere on the dashed circle to indicate their initial orientation estimate. Next, an ellipse would appear whose orientation lined up with where the subject had clicked the circle. The subject could then adjust the orientation by moving the mouse and submit the response by clicking the left mouse button. The inter-trial time was $700 \mathrm{~ms}$.

Gamified conditions. To gamify the task, we extended it with two of the most commonly used concepts in gamification (Hamari et al., 2014): points and levels. We equated the level of the "game" with the set size, i.e., the number of elements in the stimulus display. Subjects started at level 1 and would level up to the next set size each time they had accumulated 1,500 points ${ }^{2}$. On each trial, they earned or lost points based on the accuracy of their response. The scoring function was a two-part linear function in which the minimum error $\left(0^{\circ}\right)$ mapped to 100 points and the maximum error $\left(90^{\circ}\right)$ to -100 points. The error magnitude that mapped to 0 points, referred to as $E_{0}$, differed between the four gamified conditions $\left(E_{0}=20,30,45,60\right)$ and determined the difficulty of the task: the higher $E_{0}$, the easier it was to score points (Figure 1B). We expected that task difficulty might influence intrinsic motivation levels (e.g., lower motivation when the task was considered too easy or too hard). Throughout the experiment, a progress bar was visible at the top of the screen to indicate how far or close the subject was to reaching the next level (Figure 1A). After each trial, the subject received feedback about the number of gained or lost points and saw the progress bar grow or shrink accordingly. Visualizing subjects' competence may be expected to further increase their intrinsic motivation for performing the task (Ryan \& Deci, 2000). The

${ }^{2}$ More precisely, the level was computed at the beginning of each trial as $\left\lfloor\frac{\text { score }}{1500}\right\rfloor+1$, where the operation rounds a number down to the closest integer 
gamified conditions consisted of two rounds, each starting at level 1 and lasting 30 minutes. Having two rounds allowed us to examine learning effects and to dissociate these effects from potential other effects. After each round, subjects were told on the screen that they could either "Perform an additional 20 trials to improve your score" or "Skip the extra trials". The choice that subjects made here was used as "free choice" measure of motivation (Deci, 1971, 1972).

Control condition. In the control condition, no information about leveling or scores was present on the screen or in the instructions. Instead of leveling up based on performance, subjects progressed from one set size to another after a fixed amount of time. Specifically, they spent 178, 204, 260, 354, 370, and 436 seconds on set sizes 1, 2, ., and 6, respectively. These durations were chosen to match the mean duration that subjects in the $E_{0}=45^{\circ}$ gamified group spent on the same set sizes. Hence, the trial progression in the control condition roughly matched the trial progression in one of the gamified conditions, but without the presence of any gamified elements. Just as in the gamified conditions, subjects selected at the end of each run whether they wanted to perform 20 extra trials, but without a mentioning of score improvement.

\subsection{Procedure}

Subjects completed the entire experiment in a single session of approximately 90 minutes. After receiving general information about the experiment and signing an informed consent form, they received specific instructions about the task. Subjects in the gamified conditions were told that they would play two rounds of a memory game with the goal to proceed to a level as high as possible. Subjects in the control group were only told that they were going to perform two runs of a memory task with set sizes 1 to 6 . Thereafter, the subject performed five practice trials. Control subjects performed the practice trials at set size 1, while subjects in the gamified group performed the trials starting at level $=2.94$, to demonstrate the concept of earning points and leveling up. After completing the practice trials, the experiment leader would leave the room and the subject would start the first round of the experiment. After the first round, there was a short break and the subject would start the next round without intervention of the experiment leader. After finishing the second round, the experiment leader would return to the room and conduct a questionnaire, which we describe next. 


\subsection{Questionnaire}

We designed a custom questionnaire to obtain insight into aspects related to a subject's motivation (a copy of it can be found at https://osf.io/gb2kd/). The first part consisted of items similar to the ones found in the Intrinsic Motivation Inventory (IMI; McAuley, Duncan, \& Tammen, 1989; Ryan, 1982), such as "I found it interesting" and "It was important for me to perform well". These items measured motivation in four categories: Interest (items 1, 3, 6, and 8), Perceived Competence (items 5 and 9), Pressure/Tension (items 4 and 10), and Effort/Importance (items 2, 7, and 11). All items were rated on a 1 to 7 integer scale. The second part of the questionnaire consisted of items probing the subject's mood ("bored", "frustrated", etc.) in relation to different set sizes. On hindsight we found no use for the data from the second part and did not include them in the analyses.

\subsection{Analysis methods}

We analyzed the data using Bayesian statistics (Etz \& Vandekerckhove, 2018; Wagenmakers, Love, \& Marsman, 2018; Wagenmakers, Marsman, et al., 2018). All tests were performed using the JASP software package (JASP Team, 2019) with default prior settings. The subscripts of the Bayes Factors that we report indicate which test was used: $\mathrm{BF}_{10}$ indicates the probability of the data under the alternative hypothesis relative to the probability of the data under the null hypothesis; $\mathrm{BF}_{+0}$ indicates the probability of the data under the hypothesis that group 1 has a larger mean than group 2, relative to the probability of the data under the null hypothesis; $\mathrm{BF}_{\text {incl }}$ indicates the probability of the data under models that includes a main effect of the specified factor relative to the probability of the data under models that do not include this main effect. We use the scale provided in Table 1 of Wagenmakers, Love et al. (2018) for interpretation of the strength of evidence ("weak", "moderate", etc).

\subsection{Results}

2.8.1. Analysis of motivation scores. We first assess whether gamification affected selfreported scores in the motivation categories Interest, Perceived Competence, Pressure/Tension, and Effort/Importance. For each subject, we compute a single score for each category by averaging across all items within that category. We find that on average, subjects in the gamified conditions reported higher scores in all categories than control subjects (Figure 2). Bayesian t-tests reveal 
192

193

194

195

196

197

198

199

200

201

202

203

204

205

206

207

208

209

210

211

212

213

extremely strong evidence for a difference in the category of Interest $\left(\mathrm{BF}_{+0}=172\right)$, strong evidence in the category of Perceived Competence $\left(\mathrm{BF}_{+0}=88.8\right)$, and moderate evidence in the category of Effort/Importance $\left(\mathrm{BF}_{+0}=3.19\right)$. In the category of Pressure/Tension, there was weak evidence in favor of the null hypothesis $\left(\mathrm{BF}_{10}=0.32\right)$. Based on Bayesian ANOVAs with task difficulty as a fixed factor and subject as a random factor, we find for none of the motivation categories evidence that task difficulty affects the self-reported motivation scores (Interest: $\mathrm{BF}_{10}=0.13$; Perceived Competence: $\mathrm{BF}_{10}=0.28 ;$ Pressure/Tension: $\mathrm{BF}_{10}=0.64 ;$ Effort/Importance: $\left.\mathrm{BF}_{10}=0.18\right)$. In summary, these data suggest that subjects in the gamified conditions found the task more interesting, felt more competent, and possibly put more effort into it than control subjects.
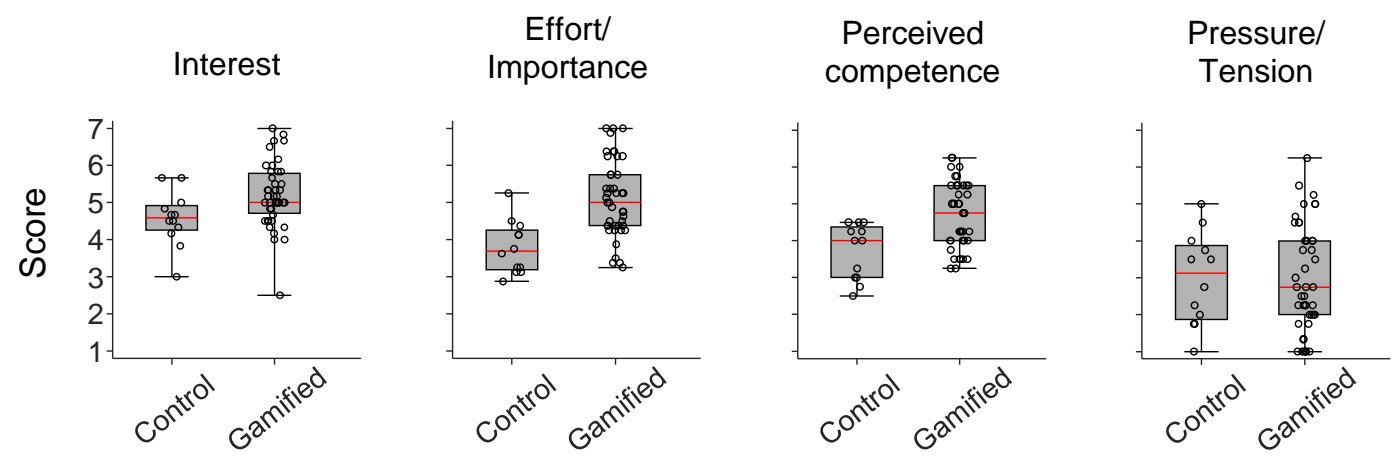

Figure 2 | Motivation scores in Experiment 1. Average self-reported scores on the questionnaire, split by motivation category. The boxes indicate the $25 \%$ and $75 \%$ quantiles, the red line the median, and the whiskers the most extreme values. The circles indicate scores of individual subjects.

2.8.2. Effect of task difficulty on VWM performance. Before we compare performance in the gamified conditions with performance in the control condition, we examine whether there is a difference in average performance between the four gamified conditions. To this end, we perform a Bayesian repeated-measures ANOVA with condition number as a between-subject factor, set size as a repeated measure, and the circular variance of the estimation error as the dependent variable. To avoid the results being affected by "survivor bias", we restrict all analyses of performance to set sizes for which we have at least 15 measurements for each subject, i.e., set sizes 1 to 4 (at higher set sizes, we lack data for at least one subject in the more difficult conditions). The result of this test provides moderate evidence for the null hypothesis that there is no difference in average performance between the gamified conditions $\left(\mathrm{BF}_{\mathrm{incl}}=0.22\right)$. Since both the motivation 
214 scores and VWM performance seem unaffected by task difficulty, we treat the four gamified

215 conditions as a single group in the remaining analyses.

A - Control condition $\quad$ Gamified conditions
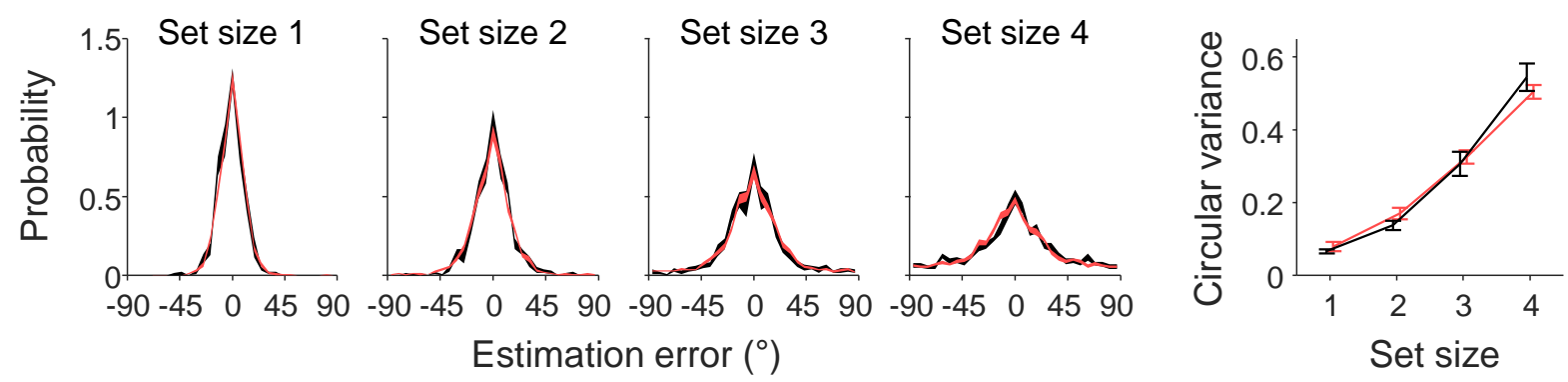

B

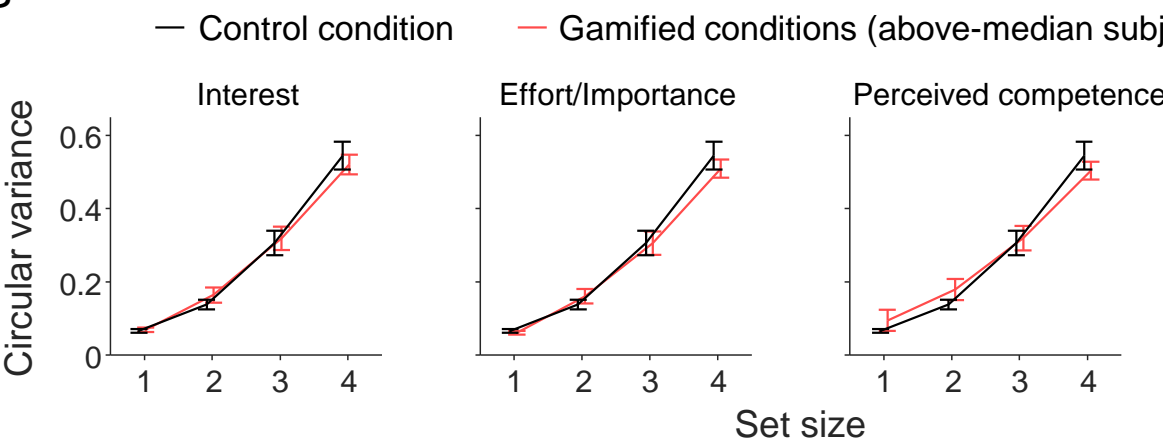

C - Below-median subjects - Above-median subjects
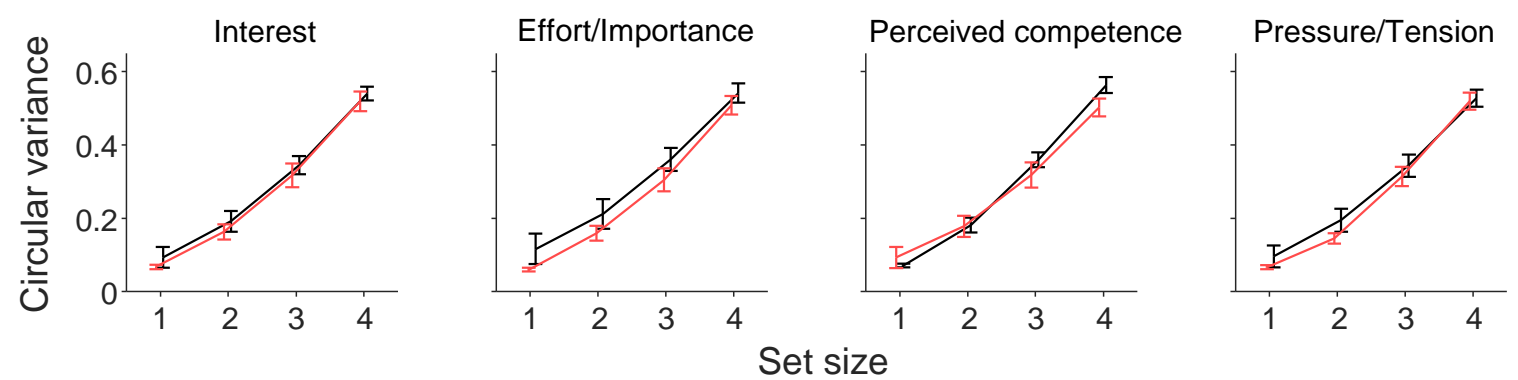

Figure 3 | VWM performance in Experiment 1. (A) Estimation error distributions averaged across subjects in the control condition (black) and subjects in the gamified conditions (red). The width of the curve indicates one standard error. The graph on the right summarizes the histograms by their circular variance. (B) Circular variance compared between subjects in the control condition and subjects with above-median motivation in the gamified conditions. The median split was performed separately for each motivation category. (C) Circular variance compared between below-median and above-median subjects in the gamified conditions. 

motivation differences between the gamified conditions and the control condition are accompanied by differences in VWM performance. The error histograms suggest that this is not the case, because they look virtually identical between subjects in the gamified conditions and subjects in the control condition (Figure 3A). Indeed, a Bayesian repeated-measures ANOVA with set size as a repeated measure, gamification as a binary between-subjects factor, and the circular variance of the error as the dependent measure reveals moderate evidence in favor of the null hypothesis of there being no effect of gamification on VWM performance $\left(\mathrm{BF}_{\text {incl }}=0.29\right)$.

One possible weakness of the previous analysis is that many subjects in the gamified group had motivation scores similar to the control subjects (Figure 2), which suggests that gamification increased motivation for only part of the subjects. Therefore, a more powerful analysis may be achieved by comparing control subjects with only the highly motivated subjects in the gamified conditions. To this end, we divide subjects in the gamified conditions into below and abovemedian groups based on the motivation scores. We find no evidence that subjects with an abovemedian Interest score performed on average differently from the control subjects $\left(\mathrm{BF}_{\text {incl }}=0.30\right)$. We neither find evidence for an effect when dividing subjects based on a median split in any of the other three motivation categories (Effort/Importance: $\mathrm{BF}_{\text {incl }}=0.28$; Pressure/Tension: $\mathrm{BF}_{\text {incl }}=0.27$; Perceived Competence: $\mathrm{BF}_{\text {incl }}=0.31$ ). Finally, we compare the above-median subjects in the gamified conditions with below-median subjects (Figure 3C). Again, regardless of which motivation category we use to make the median split, we find no evidence for a difference in VWM performance between the two groups (Interest: $\mathrm{BF}_{\text {incl }}=0.26$; Effort/Importance: $\mathrm{BF}$ incl $=0.37$; Pressure/Tension: $\mathrm{BF}_{\text {incl }}=0.26$; Perceived Competence: $\mathrm{BF}_{\text {incl }}=1.06$ ). subjects who voluntarily chose to perform additional trials at the end of a round performed better than subjects who chose not to do so. A total of 41 subjects chose to decline the option in both

245 We perform a Bayesian repeated-measures ANOVA with set size as a within-subject factor, the number of extra blocks that the subject performed as a between-subject factor, and circular

247 variance as the dependent variable. The result reveals moderate evidence against an effect of the number of extra block $\left(\mathrm{BF}_{\text {incl }}=0.12\right)$. Hence, even if subjects who voluntarily chose to perform 
additional trials were more motivated, this was not accompanied by an increase in performance on the VWM task.

2.8.5. Effect of level progress on VWM performance. So far, we have examined whether there are between-subject differences in performance based on several motivation measures. Next, we assess whether there are any within-subject effects, based on how far a subject had progressed in a level. It could be, for example, that subjects try extra hard when they are close to leveling up or down. For this analysis, we divide the data of subjects in the gamified conditions into 5 bins, with the first bin containing all trials during which the level progress bar was $0-20 \%$ filled, the second bin containing all trials during which the bar was filled $20-40 \%$, etc. As before, we only include data from set sizes 1 to 4. A Bayesian ANOVA with bin as a within-subject factor reveals very strong evidence for the null hypothesis of there not being an effect $\left(\mathrm{BF}_{\text {incl }}=0.030\right)$.

2.8.6. Effect of round on VWM performance and motivation. Finally, we examine whether there are any differences in motivation or performance between the two experiment rounds. In the control group, scores on Interest, Effort/Importance, and Perceived Competence dropped by $1.31 \pm 0.23,1.31 \pm 0.20,1.00 \pm 0.26$ points, respectively. The score on Pressure/Tension was similar in both rounds (Figure 4A, left). Consistent with the visual impression, Bayesian paired t-tests provide evidence for a drop in the first three categories $\left(\mathrm{BF}_{+0}=432,1.08 \cdot 10^{3}\right.$, and 33.7, respectively), but not in the last one $\left(\mathrm{BF}_{+0}=0.23\right)$. To test whether the differences in motivation scores is accompanied by a difference in VWM performance, we perform a two-way Bayesian ANOVA with set size and round number as independent variables and circular variance as the dependent variable. We find no evidence for a difference of round number on VWM performance $\left(\mathrm{BF}_{\text {incl }}=0.83\right.$; Fig $4 \mathrm{~B}$, left $)$. Indeed, averaged across all control subjects and set sizes, the relative difference in circular variance between the two rounds is nearly zero (they performed $1.1 \% \pm 7.2 \%$ better in the second round).

In the group of subjects who performed the gamified version of the experiment, there was no evidence for a drop in Perceived Competence $\left(\mathrm{BF}_{+0}=0.14\right)$ or Pressure/Tension $\left(\mathrm{BF}_{+0}=0.12\right)$ between the first and second round. However, consistent with the control group, there was evidence for a drop in both Interest and Effort/Importance $\left(\mathrm{BF}_{+0}=78.6\right.$ and 48.1 , respectively),

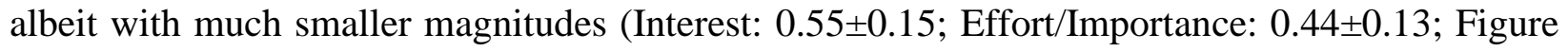
4B). Interestingly, in contrast to the control group, we find strong evidence for an effect of round on VWM performance in the gamified group $\left(\mathrm{BF}_{\text {incl }}=2.35 \times 10^{5}\right)$. Further analysis reveals that the 
circular variance of the error was $8.5 \pm 4.3 \%$ lower in the second round compared to the first round, indicating an improvement in performance (Figure 4B, right). Since this effect is opposite in direction from what one would expect as a result of the drops observed in Interest and Effort/Importance, we believe that it is best interpreted as a learning effect.

In summary, the results of the comparison between rounds suggest that gamification helps to keep subjects more interested and engaged in the task over a longer period of time. Moreover, we find indications of a learning effect in the gamified conditions, but not in the control condition. One potential explanation is that the sustained engagement of subjects in the gamified tasks was beneficial for learning. However, the lack of a learning effect in the control condition may just as well have been due to lower statistical power (only 12 subjects compared to 50 in the gamified conditions). Most importantly, consistent with the previous analyses, we find no evidence that higher motivation is accompanied by better VWM performance.

A

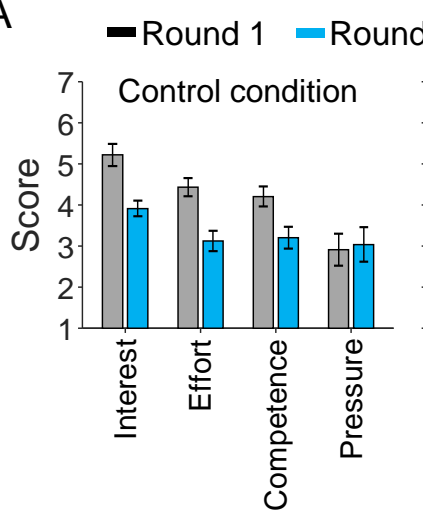

B

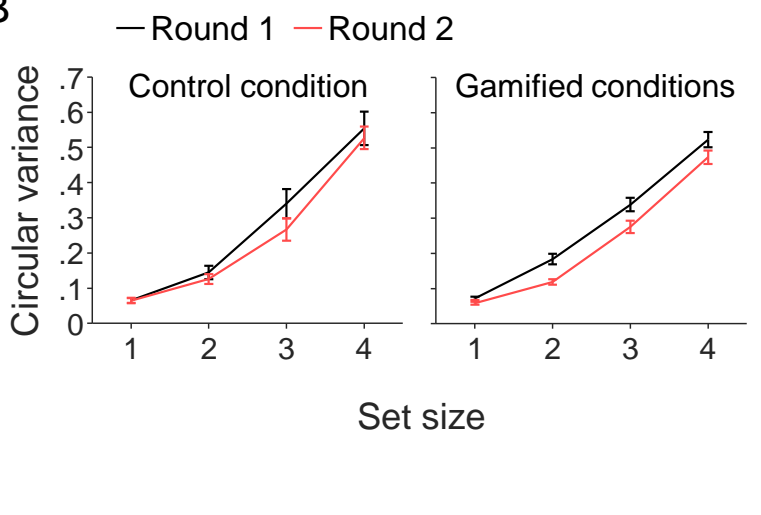

Figure 4 | Motivation and performance differences between rounds in Experiment 1. (A) Difference in self-reported motivation scores between the two rounds. (B) Difference in circular variance of the estimation error distribution between the two rounds.

Gamified conditions

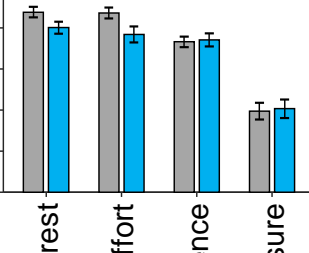

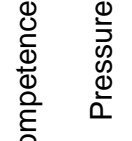

\subsection{Discussion}

We gamified a standard VWM task and measured how this affected people's motivation and performance. While the questionnaire data suggest that subjects in gamified conditions had higher motivation than subjects in the control condition, we did not find any evidence for a difference in memory performance. This null effect on performance is consistent with earlier indications that VWM performance is not sensitive to monetary incentives (van den Berg, Zou, \& Ma, 2019; Zhang \& Luck, 2011). 
The perhaps most straightforward explanation for the absence of an effect is that the total amount of invested VWM resource may be fixed, meaning that no matter how hard subjects try, they cannot improve their performance. This would be somewhat surprising, however, because such inflexibility stands in stark contrast to the flexibility that has been found in how subjects distribute VWM resources across a given set of items: when certain items have higher associated reward (Morey, Cowan, Morey, \& Rouder, 2011) or are more likely to be probed than others (Bays, 2014; Emrich et al., 2017; Yoo, Klyszejko, Curtis, \& Ma, 2018), subjects assign more resources to that item compared to the other ones. Moreover, it has been found that subjects can increase performance on cued items without a cost for uncued items (Myers, Chekroud, Stokes, \& Nobre, 2018) and that specific forms of feedback can improve overall performance on VWM tasks (Adam \& Vogel, 2016). Both those findings suggest that it is possible to induce a net increase in utilized VWM resources through experimental manipulations. Also, investing a fixed amount of total resource regardless of the task is suboptimal from a resource-rationality perspective (Van den Berg \& Ma, 2018).

An alternative explanation of the null effect is that our experimental design might not have been suitable for inducing or detecting motivation-related flexibility in VWM resource investment. One potential problem is that we used very short stimulus times (200 milliseconds), which may have led to incomplete encoding of the items (Bays et al., 2009). As a result, the maximum precision in VWM was possibly limited by the quality of the input rather than by the amount of available VWM resources. Moreover, it is possible that motivation manipulations are only effective when they are administered on a trial-by-trial basis, as suggested by an earlier study on the relation between task preparation and reward (Shen \& Chun, 2011). To address these potential weaknesses in the experimental design, we perform a second experiment with a longer stimulus presentation time and a within-subject manipulation of motivation.

\section{EXPERIMENT 2}

\subsection{Participants}

A total of 12 participants with self-reported normal or corrected-to-normal vision were recruited using posters at various campuses of Uppsala University ( 8 females; age mean \pm s.e.m. = 27.6 \pm 1.39 ). The study was approved by the Regional Ethical Review Board in Uppsala and 
conducted according to the Declaration of Helsinki Principles. All participants signed informed consent and received a cinema voucher with a value of approximately $\$ 12$ for their participation. One of the participants (S6) had earlier participated as a control subject in Experiment 1.

\subsection{Experiment procedure}

The methods of Experiment 2 were identical to those of Experiment 1, except for the following differences. Most importantly, each subject was tested under three different scoring function with varying amounts of maximum reward (Figure 5B). The scoring functions were of the form score $=A \exp \left(-\frac{\varepsilon^{2}}{2 \sigma^{2}}\right)$, where $\varepsilon$ is the estimation error in degrees, $A$ determines the maximum score (obtained when $\varepsilon=0$ ), and $\sigma$ determines how quickly the score declines as a function of the error. The "low reward" function always gave 7 points, regardless of the accuracy of the subject's response $(A=7, \sigma=\infty)$, the "medium reward" function gave a score between 0 and $21(A=21, \sigma=30)$, and the "high reward" function gave a score between 0 and $101(A=101, \sigma=20)$. Subjects were told prior to the experiment that there were trials with low, medium, and high potential reward. However, to avoid that they would challenge themselves to always score the maximum number of points - regardless of the scoring function - we did not tell them the maximum score associated with each function. Within each block of 15 trials, each scoring function was used five times, presented in a random order. To inform the subjects about the reward level of the upcoming trial, we added a reward cue $(1.1 \mathrm{sec})$ at the start of the trial (Figure 5A).

We made several other minor changes to the experiment. First, we increased the stimulus presentation time from 200 to $900 \mathrm{~ms}$, to reduce the risk that memory quality is limited by the quality of the sensory input rather than the availability of VWM resources. Second, to collect more trials per set size, we increased the number of points required for levelling up from 1,500 to 1,750. Third, we made a minor improvement in how we controlled for fixation errors. In addition to ensuring that subjects were fixating at the start of the trial, we now also forced them to keep fixating during the stimulus presentation and memory delay period. If they broke fixation ${ }^{3}$ during this period, the trial would be terminated with a message "Invalid trial. Please fixate at the center when the stimulus is shown" or "Invalid trial. Please fixate at the center until the response stage".

\footnotetext{
${ }^{3}$ We considered fixation to be broken once the eye tracker returned 5 measurements in which the gaze location was farther than 150 pixels away from the center of the fixation dot.
} 
361 The time spent on invalid trials was added to the round time, such that each subject spent 30

362 minutes on valid trials, regardless of the number of invalid trials. Fourth, we added a 30-second

363 forced break between set sizes; after those 30 seconds, subjects could resume the experiment by a

364 keypress whenever they felt ready. Finally, we increased the number of practice trials from 5 to 7

365 to demonstrate what would happen when breaking of fixation.

A

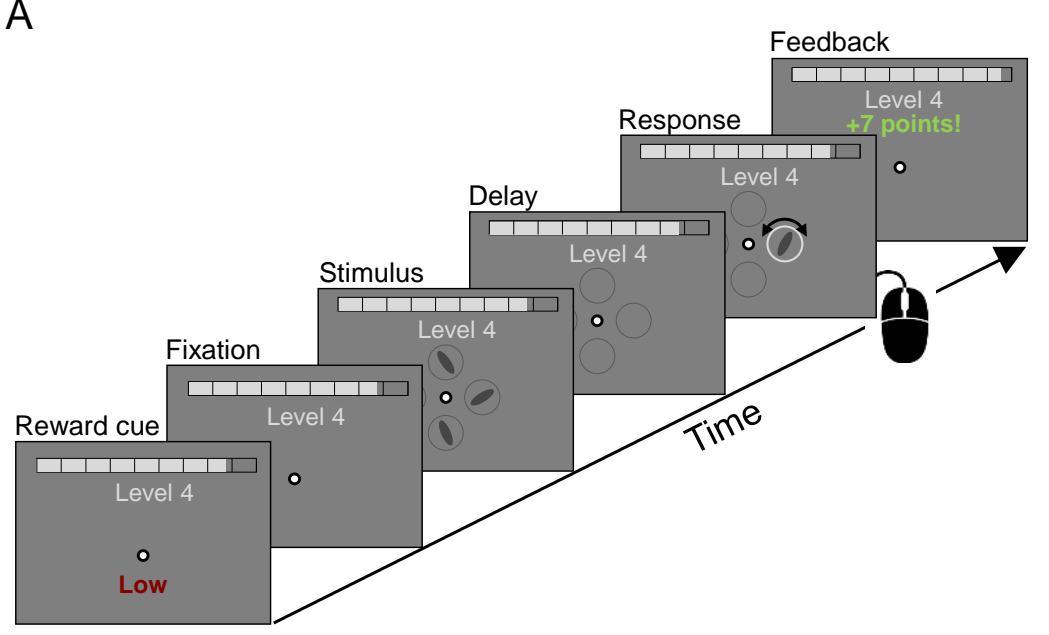

B

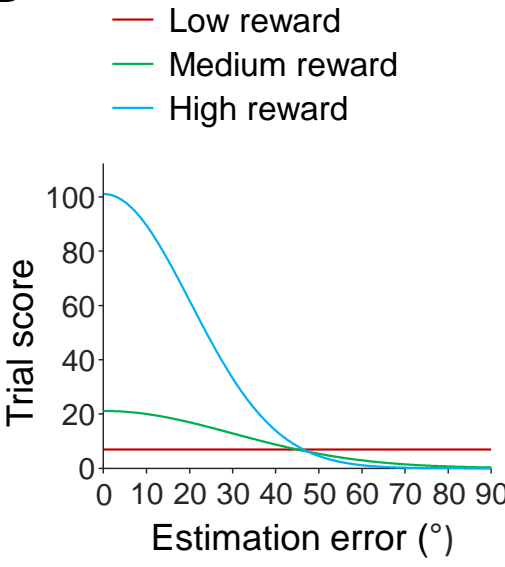

C

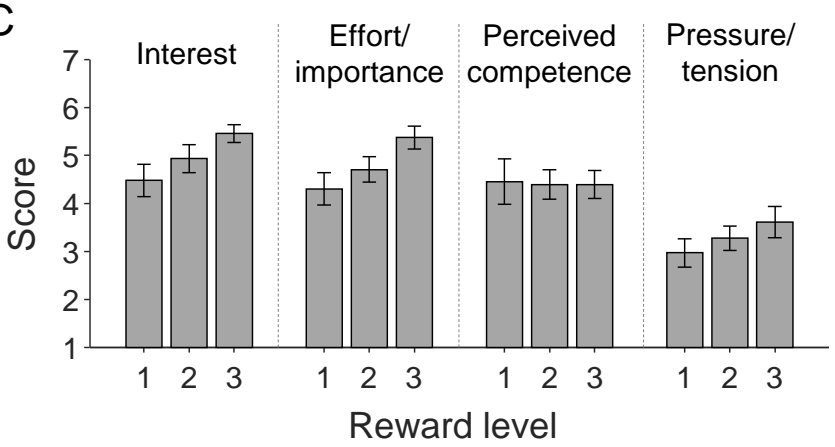

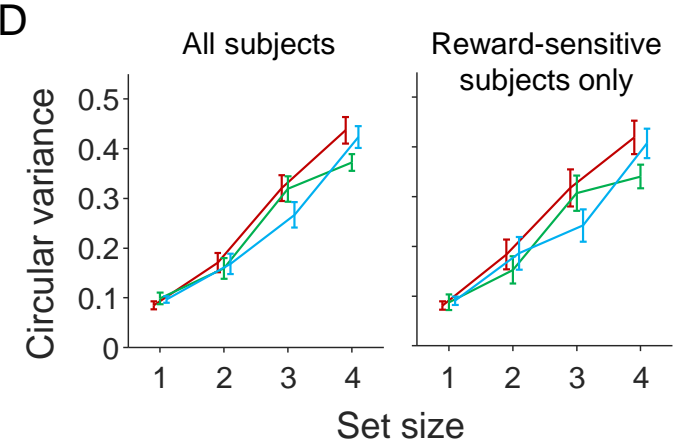

Figure 5 | Design and results of Experiment 2. (A) Schematic illustration of a single experimental trial. (B) Scoring functions used in the three trial types. (C) Motivation scores split by category and reward level. (D) Circular variance of the estimation error distribution split by reward level (see panel B for legend), plotted separately for all subjects (left) and for the group of subjects who reported higher motivation for high-reward trials (right).

\subsection{Questionnaire}

After finishing the experiment, subjects filled out a questionnaire in a web browser. The questions were the same as in the first part of the questionnaire used in Experiment 1, except that 
372 a few items were added in each of the four categories (again based on IMI). For all the items we

373 asked separate ratings for low, medium, and high reward trials (a copy of the questionnaire can be

374 found at https://osf.io/gb2kd/). Items 1, 3, 6, 8, 13, and 15 were used to compute Interest scores,

375 items 2, 7, 12, and 17 to compute Effort/Importance scores, items 5, 9, 14, and 16 to compute

376 Perceived Competence scores, and items 4, 10, 11 to compute Pressure/Tension scores. The second

377 part of the questionnaire of Experiment 1 was not included.

378

379

380

381

382

383

384

385

386

387

388

389

390

391

392

393

394

395

396

397

398

399

400

401

\subsection{Results}

A visual inspection of the questionnaire data (Figure 5C) suggests a positive relationship between the reward level (low, medium, high) of a trial and the average motivation score in the categories Interest, Effort/Importance, and Pressure/Tension. To test this statistically, we perform a Bayesian linear regression with Reward Level (coded as 1, 2, or 3 ) as a covariate and motivation score as the dependent variable. We find moderate evidence for a positive relation in categories Interest $\left(\mathrm{BF}_{10}=5.03 ; 95 \%\right.$ credible interval on the coefficient: [0.00, 0.46]) and Effort/Importance $\left(\mathrm{BF}_{10}=3.36,95 \%\right.$ credible interval on the coefficient: $\left.[0.00,0.70]\right)$, but not in categories Perceived Competence $\left(\mathrm{BF}_{10}=0.32\right)$ and Pressure/Tension $(\mathrm{BF} 10=0.82)$. Hence, on average, subjects apparently found the task more interesting at high-reward trials compared to low-reward trials and were willing to put more effort into those trials.

We next examine whether the effect of reward level on motivation was accompanied by an effect on VWM performance (Figure 5D, left). We perform a Bayesian ANOVA with circular variance of the error distribution as the dependent variable, set size and reward level as fixed factors, and subject number as a random factor. Just as in the analyses of Experiment 1, we only include set sizes 1 to 4 . The results provide strong evidence for the null hypothesis that there is no effect of reward level on the circular variance $\left(\mathrm{BF}_{\text {incl }}=0.091\right)$.

Closer inspection of the questionnaire data reveals that the increase of Interest and Effort/Importance with reward level exists for only 7 of the 12 subjects. The remaining 5 subjects either had a decreasing or non-monotonic pattern in one of the two motivation categories. This indicates that only 7 subjects may have been sensitive to the reward manipulation. To verify that the null result on VWM performance was not due to inclusion of the other 5 subjects in our analysis, we rerun the ANOVA on only the 7 subjects with consistent motivation score patterns 
(Figure 5D, right). We again find evidence for the null hypothesis that there is no effect of reward level on VWM performance $\left(\mathrm{BF}_{\text {incl }}=0.17\right)$.

Another reason for the null result at the group level could be that there is heterogeneity in the effect direction across subjects, such that the effects cancel each other out (e.g., some subjects may have performed better with higher reward, while others might have performed worse). To examine this, we perform a model comparison at the level of individual subjects. The first model is the standard version of the variable-precision model (van den Berg, Awh, \& Ma, 2014; van den Berg et al., 2012), which has three free parameters: $\bar{J}_{1}$ (encoding precision at set size 1 ), $\tau$ (controlling the amount of variability in resource allocation), and $\alpha$ (controlling how encoding precision changes with set size). The relation between set size, $N$, and encoding precision, $\overline{\boldsymbol{J}}$, is defined as $\bar{J}(N)=\bar{J}_{1} N^{\alpha}$. We use 5-fold cross validation to compare the fit of this model with a variant in which encoding precision depends on both set size and reward level $R$ (coded as 1,2 , and 3): $\bar{J}(N)=\bar{J}_{1} N^{\alpha} R^{\beta}$, where $\beta$ is an additional free parameter. When $\beta=0$, the two models are identical. For subjects with a strong relation between reward level and VWM performance, $\beta=0$ will not provide a good fit. For those subjects we should find a strong advantage of the second model over the first one. By contrast, we find that for every subject the cross-validated log likelihood difference between the two models is close to zero (range: 1.05 in favor of the first model to 1.99 points in favor of the second model). In fact, for 10 of the subjects the first model is favored. These results corroborate the earlier evidence suggesting that VWM performance was unaffected by reward level.

\subsection{Discussion}

The purpose of Experiment 2 was to address two potential weaknesses in Experiment 1 by increasing the stimulus presentation time and manipulating motivation on a trial-by-trial basis. The post-questionnaire data of Experiment 2 showed a positive relation between Interest and Effort/Important scores on the one hand and reward level on the other hand, indicating that our within-subject design had the desired effect on motivation. However, the increase in motivation on high-reward trials was not accompanied by an increase in VWM performance. Hence, the results of Experiment 2 are consistent with those of Experiment 1: gamification had a positive effect on motivation, but left performance unaffected. 


\section{GENERAL DISCUSSION}

While it is known that gamification can increase subjects' intrinsic motivation, enjoyment, engagement, and perceived competence in a task (Hamari et al., 2014), little is known about effects of gamification on cognitive performance. Here, we performed two experiments to investigate whether gamification of a standard VWM task improves subjects' performance on that task. Consistent with previous literature, we found positive effects of gamification on subjects' selfreported interest in the task and the amount of effort they reportedly put into it. However, in neither experiment did we find any differences in VWM performance. We thus conclude that gamification can make people more motivated to perform VWM tasks, but it does not necessarily make them better at it.

Our finding that there was no relation whatsoever between motivation and VWM performance in our experiments is puzzling for several reasons. First, there is convincing evidence that people are able to flexibly assign more VWM resources to important items compared to less important ones, in a way that seemingly optimizes performance (Bays, 2014; Emrich et al., 2017; Morey et al., 2011; Yoo et al., 2018). As we have demonstrated earlier (van den Berg \& Ma, 2018), it would be suboptimal if people would always invest the same amount of VWM, independent of task properties. Our finding that they nevertheless seemed to do so in our experiments raises the question why VWM has evolved to be approximately optimal in distributing a given amount of resource across items, but at the same time uses a highly suboptimal policy to determine the total amount of VWM resource to invest in a task. Moreover, it is known that motivation and expected reward affect dopamine concentration (e.g., Di Chiara, 2005; Hamid et al., 2015; Syed et al., 2015) and that dopamine concentration, in turn, is related to VWM performance (e.g., Arnsten, 1998; Ashby \& Valentin, 2017; Cools et al., 2008; Okimura et al., 2015; Sawaguchi \& Goldman-Rakic, 1991; Williams \& Goldman-Rakic, 1995). Based on those findings, one would expect - contrary to our findings - that any experimental manipulation that affects participants' motivation, also affects their VWM performance.

An alternative explanation for our null findings is that subjects may have been overperforming: regardless of the condition they were in, they may have felt a responsibility to perform as well as they could, for instance as a justification for their payment, out of a desire to deliver high-quality data to the researchers, or out of fear to be confronted with their performance after finishing the experiment. Based on informal feedback, we know that at least one of the 
subjects who voluntarily performed the additional trials did so because she believed it would help the researchers. While most studies that manipulate motivation do so by incentivizing subjects, if overperformance is a real issue, it might be interesting for future studies to look into ways to decrease motivation levels. Moreover, it may be that highly artificial stimuli are not very suitable for manipulating motivation levels, for instance due to a lack of engagement in the task. Therefore, it might also be fruitful if future studies would use more naturalistic stimuli. Another potential limitation of the present study is that we used a questionnaire to measure how motivated subjects were and how much effort they put into the task. Another limitation of the present study is that we only analyzed VWM performance at relatively low set sizes (1 to 4). We excluded higher set sizes in order to avoid "survivor bias" effects in the results. However, the subjects" answers to the questionnaire items that we used to measure intrinsic motivation were based on their experience of all set sizes they performed. Hence, the motivation data may be unreliable for the set sizes that we used to analyze VWM performance differences. To address this problem, it would have been good to complement the self-report measures with pupillary dilation data, which is known to correlate with attention and effort (Hoeks \& Levelt, 1993; van der Wel \& van Steenbergen, 2018). In the present study we collected eye movement data, including pupil dilation measurements, but we did so mainly to verify that subjects were fixating and not with the aim to use them in our analyses. As a result, the collected data are unfortunately unsuitable for analysis.

As a final remark, we believe that our study may serve as a showcase of open science in a field that is currently plagued by concerns about replicability (Aarts et al., 2015) . Although an increasing number of authors make their data available - partly driven by changes in journal policies - researchers still seem to be wary of publishing null results, especially when this a result contradicts their own theory. Keeping such findings hidden in a file drawer may have short-term benefits for the researchers, but is seen as one of the major factors behind the replicability crisis.

\section{ACKNOWLEDGEMENTS}

RvdB is supported by Grant 2018-01947 from the Swedish Research Council (Vetenskapsrådet) and Grant RIK17-1157:2 of the Bank of Sweden Tercentenary Foundation (Stiftelsen Riksbankens Jubileumsfond). The funding sources had no role in the study design, the collection, analysis and interpretation of data, the writing of the report, and the decision to submit the article for publication. 


\section{DECLARATION OF INTEREST}

495

496

497

498

499

500

501

502

503

504

505

506

507

508

509

510

511

512

513

514

515

516

517

518

519

520

521

522

523

524

None.

\section{REFERENCES}

Aarts, A. A., Anderson, J. E., Anderson, C. J., Attridge, P. R., Attwood, A., Axt, J., ... Zuni, K. (2015). Estimating the reproducibility of psychological science. Science. https://doi.org/10.1126/science.aac4716

Adam, K. C. S., \& Vogel, E. K. (2016). Reducing failures of working memory with performance feedback. Psychonomic Bulletin \& Review, 23(5), 1520-1527. https://doi.org/10.3758/s13423-016-1019-4

Arnsten, A. F. T. (1998). Catecholamine modulation of prefrontal cortical cognitive function. Trends in Cognitive Sciences. https://doi.org/10.1016/S1364-6613(98)01240-6

Ashby, F. G., \& Valentin, V. V. (2017). Multiple systems of perceptual category learning: Theory and cognitive tests. In Handbook of categorization in cognitive science, 2nd ed. (pp. 157-188). https://doi.org/10.1016/B978-0-08-101107-2.00007-5

Attwell, D., \& Laughlin, S. B. (2001). An energy budget for signaling in the grey matter of the brain. Journal of Cerebral Blood Flow and Metabolism : Official Journal of the International Society of Cerebral Blood Flow and Metabolism, 21(10), 1133-1145. https://doi.org/10.1097/00004647-200110000-00001

Bays, P. M. (2014). Noise in neural populations accounts for errors in working memory. The Journal of Neuroscience: The Official Journal of the Society for Neuroscience, 34(10), 3632-3645. https://doi.org/10.1523/JNEUROSCI.3204-13.2014

Bays, P. M., Catalao, R. F. G., \& Husain, M. (2009). The precision of visual working memory is set by allocation of a shared resource. Journal of Vision, 9(10), 7.1-11. https://doi.org/10.1167/9.10.7

Brady, T. F., Konkle, T., \& Alvarez, G. A. (2011). A review of visual memory capacity: Beyond individual items and toward structured representations. Journal of Vision, 11(5), 4. https://doi.org/10.1167/11.5.4

Cools, R., Gibbs, S. E., Miyakawa, A., Jagust, W., \& D’Esposito, M. (2008). Working memory capacity predicts dopamine synthesis capacity in the human striatum. The Journal of Neuroscience: The Official Journal of the Society for Neuroscience, 28(5), 1208-1212. 
https://doi.org/10.1523/JNEUROSCI.4475-07.2008

Deci, E. L. (1971). Effects of externally mediated rewards on intrinsic motivation. Journal of Personality and Social Psychology. https://doi.org/10.1037/h0030644

Deci, E. L. (1972). Intrinsic motivation, extrinsic reinforcement, and inequity. Journal of Personality and Social Psychology. https://doi.org/10.1037/h0032355

Deterding, S., Dixon, D., Khaled, R., \& Nacke, L. (2011). From game design elements to gamefulness: Defining “gamification.” Proceedings of the 15th International Academic MindTrek Conference: Envisioning Future Media Environments, MindTrek 2011. https://doi.org/10.1145/2181037.2181040

Di Chiara, G. (2005). Chapter VI Dopamine, motivation and reward. Handbook of Chemical Neuroanatomy. https://doi.org/10.1016/S0924-8196(05)80010-7

Dicheva, D., Dichev, C., Agre, G., \& Angelova, G. (2015). Gamification in Education: A Systematic Mapping Study. Educational Technology \& Society, 18(3), 75-88. https://doi.org/10.1109/EDUCON.2014.6826129.

Emrich, S. M., Lockhart, H. A., \& Al-Aidroos, N. (2017). Attention mediates the flexible allocation of visual working memory resources. Journal of Experimental Psychology: Human Perception and Performance, 43(7), 1454-1465. https://doi.org/10.1037/xhp0000398

Etz, A., \& Vandekerckhove, J. (2018). Introduction to Bayesian Inference for Psychology. Psychonomic Bulletin and Review. https://doi.org/10.3758/s13423-017-1262-3

Fuster, J. M., \& Alexander, G. E. (1971). Neuron Activity Related to Short-Term Memory. Science, 173(3997), 652-654. https://doi.org/10.1126/science.173.3997.652

Hamari, J., Koivisto, J., \& Sarsa, H. (2014). Does gamification work? - A literature review of empirical studies on gamification. Proceedings of the Annual Hawaii International Conference on System Sciences. https://doi.org/10.1109/HICSS.2014.377

Hamari, J., \& Lehdonvirta, V. (2010). Game design as marketing: How game mechanics create demand for virtual goods. International Journal of Business Science and Applied Management.

Hamid, A. A., Pettibone, J. R., Mabrouk, O. S., Hetrick, V. L., Schmidt, R., Vander Weele, C. M., ... Berke, J. D. (2015). Mesolimbic dopamine signals the value of work. Nature Neuroscience. https://doi.org/10.1038/nn.4173 
Hanus, M. D., \& Fox, J. (2015). Assessing the effects of gamification in the classroom: A longitudinal study on intrinsic motivation, social comparison, satisfaction, effort, and academic performance. Computers and Education, 80, 152-161. https://doi.org/10.1016/j.compedu.2014.08.019

Hoeks, B., \& Levelt, W. J. M. (1993). Pupillary dilation as a measure of attention: a quantitative system analysis. Behavior Research Methods, Instruments, \& Computers. https://doi.org/10.3758/BF03204445

JASP Team. (2019). JASP (Version 0.10.2). [Computer Software].

Laughlin, S. B. (2001). Energy as a constraint on the coding and processing of sensory information. Current Opinion in Neurobiology, Vol. 11, pp. 475-480. https://doi.org/10.1016/S0959-4388(00)00237-3

Luck, S. J., \& Vogel, E. K. (2013). Visual working memory capacity: From psychophysics and neurobiology to individual differences. Trends in Cognitive Sciences, 17(8), 391-400. https://doi.org/10.1016/j.tics.2013.06.006

Ma, W. J., Husain, M., \& Bays, P. M. (2014). Changing concepts of working memory. Nature Neuroscience, 17(3), 347-356. https://doi.org/10.1038/nn.3655

McAuley, E., Duncan, T., \& Tammen, V. V. (1989). Psychometric Properties of the Intrinsic Motivation Inventory in a Competitive Sport Setting: A Confirmatory Factor Analysis. Research Quarterly for Exercise and Sport, 60(1), 48-58. https://doi.org/10.1080/02701367.1989.10607413

Morey, C. C., Cowan, N., Morey, R. D., \& Rouder, J. N. (2011). Flexible attention allocation to visual and auditory working memory tasks: Manipulating reward induces a trade-off. Attention, Perception, and Psychophysics. https://doi.org/10.3758/s13414-010-0031-4

Myers, N. E., Chekroud, S. R., Stokes, M. G., \& Nobre, A. C. (2018). Benefits of flexible prioritization in working memory can arise without costs. Journal of Experimental Psychology: Human Perception and Performance. https://doi.org/10.1037/xhp0000449

Nassar, M. R. M. R., Helmers, J. C. J. C., \& Frank, M. . M. J. (2018). Chunking as a rational strategy for lossy data compression in visual working memory. In Psychological Review (Vol. 125). https://doi.org/10.1037/rev0000101

Okimura, T., Tanaka, S., Maeda, T., Kato, M., \& Mimura, M. (2015). Simulation of the capacity and precision of working memory in the hypodopaminergic state: Relevance to 
schizophrenia. Neuroscience, 295, 80-89.

$$
\text { https://doi.org/10.1016/J.NEUROSCIENCE.2015.03.039 }
$$

Ryan, RM; Deci, E. (2000). Self-determination theory and the facilitation of intrinsic motivation, social development, and well-being. American Psychologist.

Ryan, R. M. (1982). Control and information in the intrapersonal sphere: An extension of cognitive evaluation theory. Journal of Personality and Social Psychology. https://doi.org/10.1037/0022-3514.43.3.450

Sawaguchi, T., \& Goldman-Rakic, P. (1991). D1 dopamine receptors in prefrontal cortex: involvement in working memory. Science. https://doi.org/10.1126/science.1825731

Shen, Y. J., \& Chun, M. M. (2011). Increases in rewards promote flexible behavior. Attention, Perception, and Psychophysics. https://doi.org/10.3758/s13414-010-0065-7

Sterling, P., \& Laughlin, S. (2015). Principles of neural design. MIT Press.

Subhash, S., \& Cudney, E. A. (2018). Gamified learning in higher education: A systematic review of the literature. Computers in Human Behavior. https://doi.org/10.1016/j.chb.2018.05.028

Syed, E. C. J., Grima, L. L., Magill, P. J., Bogacz, R., Brown, P., \& Walton, M. E. (2015). Action initiation shapes mesolimbic dopamine encoding of future rewards. Nature Neuroscience. https://doi.org/10.1038/nn.4187

van den Berg, R., Awh, E., \& Ma, W. J. W. J. (2014). Factorial comparison of working memory models. Psychological Review, 121(1), 124-149. https://doi.org/10.1037/a0035234

van den Berg, R., \& Ma, W. J. (2018). A resource-rational theory of set size effects in visual working memory. ELife, 7(e34963). https://doi.org/10.7554/eLife.34963

van den Berg, R., Shin, H., Chou, W.-C., George, R., \& Ma, W. J. (2012). Variability in encoding precision accounts for visual short-term memory limitations. Proceedings of the National Academy of Sciences, 109(22), 8780-8785. https://doi.org/10.1073/pnas.1117465109

van den Berg, R., Zou, Q., \& Ma, W. J. (2019). Does monetary reward increase visual working memory performance? BioRxiv, 767343. https://doi.org/10.1101/767343

van der Wel, P., \& van Steenbergen, H. (2018). Pupil dilation as an index of effort in cognitive control tasks: A review. Psychonomic Bulletin and Review. https://doi.org/10.3758/s13423018-1432-y 
618 Wagenmakers, E. J., Love, J., \& Marsman, M. (2018). Bayesian inference for psychology. Part

619 II: Example applications with JASP. Psychon Bull Rev, 25(1), 58-76.

620 Wagenmakers, E. J., Marsman, M., Jamil, T., Ly, A., Verhagen, J., Love, J., ... Morey, R. D.

621 (2018). Bayesian inference for psychology. Part I: Theoretical advantages and practical ramifications. Psychonomic Bulletin and Review. https://doi.org/10.3758/s13423-017-1343-

623 3

624 Wilken, P., \& Ma, W. J. (2004). A detection theory account of change detection. Journal of 625 Vision, 4(12), 1120-1135. https://doi.org/10:1167/4.12.11

626 Williams, G. V., \& Goldman-Rakic, P. S. (1995). Modulation of memory fields by dopamine D1 627 receptors in prefrontal cortex. Nature. https://doi.org/10.1038/376572a0

628 Yoo, A. H., Klyszejko, Z., Curtis, C. E., \& Ma, W. J. (2018). Strategic allocation of working 629 memory resource. Scientific Reports. https://doi.org/10.1038/s41598-018-34282-1

Zhang, W., \& Luck, S. J. (2011). The number and quality of representations in working memory. Psychological Science, 22(11), 1434-1441. https://doi.org/10.1177/0956797611417006 